\title{
Preparation and growth mechanism of nickel nanowires under applied magnetic field
}

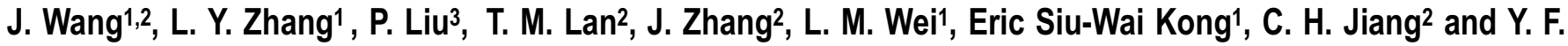 \\ Zhang1,*
}

Nickel nanowires with large aspect ratio of up to 300 have been prepared by a hydrazine hydrate reduction method under applied magnetic field. The diameter of nickel nanowires is about $200 \mathrm{~nm}$ and length up to $60 \mu \mathrm{m}$. The role of magnetic field on the growth of magnetic nanowires is discussed and a magnetic nanowire growth mechanism has been proposed. Nickel ions are firstly reduced to nickel atoms by hydrazine hydrates in a strong alkaline solution and grow into tiny spherical nanoparticles. Then, these magnetic particles will align under a magnetic force and form linear chains. Furthermore, the as-formed chains can enhance the local magnetic field and attract other magnetic particles nearby, resulting finally as linear nanowires. The formation and the size of nanowires depend strongly on the magnitude of applied magnetic field.

Keywords: Nanowire growth mechanism; Nickel nanowires; Magnetic-field assisted synthesis

Citation: J. Wang, L. Y. Zhang, P. Liu, T. M. Lan, J. Zhang, L. M. Wei, Eric Siu-Wai Kong, C.H. Jiang and Y.F. Zhang, "Preparation and growth mechanism of nickel nanowires under applied magnetic field", Nano-Micro Lett. 2, 134-138 (2010). doi: 10.5101/nml.v2i2.p134-138

Morphological control in nanostructures has become a key issue in the preparation of electronic, photonic devices as well as functional materials $[1,2]$. In addition, much attention has been focused on one-dimensional (1-D) nanostructure such as nanorods, nanowires, nanofibers and nanochains due to their potential applications in nanodevices [3-5]. As a typical magnetic material, 1-D metallic Ni nanomaterial in uniform morphology and high purity has become increasingly mandatory for specific applications such as microwave absorbing materials, magnetic recording media, gas sensors, drug deliveries, commercial batteries and catalysts [6-12]. To date, much effort has been made to prepare $\mathrm{Ni}$ nanomaterial with 1-D structure. Template methods are the most common synthesis technique. For example, Yu et al. prepared nickel nanowire arrays in alumina templates by using a chemical electrodeposition method [13]. Zhang et al. fabricated nickel hollow fiber using natural silk as the template [14]. It is well known that template-based methods are not facile and in fact, it is difficult to synthesize a large amount of nanomaterials using the template-based route [15]. In comparison, chemical reduction approach is much simpler and having lower cost. Recently Niu et al. fabricated acicular nickel nanocrystallites with an average length of $10 \mu \mathrm{m}$ and a diameter of about 200 nm, using PEG and CTAB surfactant in a sealed autoclave under an external magnetic field, under high temperature and pressure [16]. Although Gong et al. synthesized Ni nanowires under normal pressure, ethylene glycol was used as the solvent [17]. In recent years, our research group has reported the facile synthesis of Ni nanowires in aqueous ethanol solution $[18,19]$. This is a much more promising approach to synthesize magnetic nanowires in large scale since it is simple, low-cost, high yield, and environmentally friendly.

In previous work, we have reported the synthesis of $\mathrm{Ni}$ nanowires [18,19]. In this investigation, we studied the

${ }^{1}$ National Key Laboratory of Nano/Micro Fabrication Technology, Key Laboratory for Thin Film and Microfabrication of the Ministry of Education, Institute of Micro and Nano Science and Technology, Shanghai Jiao Tong University, Shanghai 200240, China

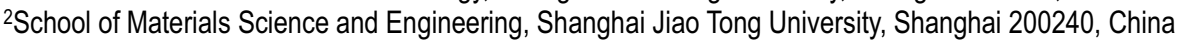

${ }^{3}$ Zhongyuan University of Technology, Zhengzhou City, Henan Province 450007, China

*Corresponding author. E-mail: yfzhang@sjtu.edu.cn; Tel.: +86(0)21-34205665; fax: +86(0)21-34205665 
magnetic field dependence of the morphology as well as the formation process of $\mathrm{Ni}$ nanowires. A possible growth mechanism has been proposed. The method reported in this paper can be used to fabricate large-scale Ni nanowires, as well as other 1-D magnetic nanowires. The proposed mechanism may further shed light on the fabrication approach on magnetic nanowires with high quality.

\section{Experimental Section}

All chemical reagents in our experiments are analytical grade and are used without further purification. Nickel chloride hexahydrate $\left(\mathrm{NiCl}_{2} \cdot 6 \mathrm{H}_{2} \mathrm{O}\right)$ was dissolved in a solution composed of distilled water and ethanol. Then, hydrazine hydrate with concentration of $85 \%$ was injected and the $\mathrm{pH}$ value was adjusted to 13.7 by using $5 \mathrm{M}$ sodium hydroxide $(\mathrm{NaOH})$ solution. The as-prepared, blue transparent solution was transferred to a flask and kept at $50^{\circ} \mathrm{C}$ for $10 \sim 30 \mathrm{~min}$. A permanent $\mathrm{NdFeB}$ magnet was placed under the flask and different applied magnetic fields can be obtained by adjusting the distance between the magnet and the flask. In this investigation, the Ni nanowires were synthesized at a magnetic field of $0.5 \mathrm{~T}$. The magnetic field was measured using a Gauss meter (Model SHT-4A). After the reaction was completed, the resulting grey-black, fluffy solid product floated on top of the solution surface and the solution became colorless and transparent. The product was collected and washed several times using distilled water and ethanol under an applied magnetic field and then dried in vacuum oven at $60^{\circ} \mathrm{C}$ for $12 \mathrm{~h}$.

The size and surface morphology of $\mathrm{Ni}$ nanowires were investigated by emission scanning electron microscopy (SEM, Zeiss Ultra 55, Germany) at an accelerating voltage of $5 \mathrm{kV}$. The detailed microstructure of the nanowires was studied by a transmission electron microscopy (TEM, JEM-100CX, JEOL, Japan). X-ray diffraction pattern was measured by a $18 \mathrm{~kW}$ advanced X-ray diffractometer (D8 ADVANCE, Bruker, Germany) with $\mathrm{Cu} K \alpha$ radiation $(\lambda=1.54056 \mathrm{~nm})$.

\section{Results and Discussion}

The role of the hydrazine hydrate as a reducing agent in the reaction was investigated. It was found that no grey-black products formed if hydrazine hydrate was absent even at high temperature of $80^{\circ} \mathrm{C}$ and strong magnetic field of $0.5 \mathrm{~T}$. This indicates that hydrazine hydrate played a very important role in the reaction as a reducing agent. Moreover, it was found that the reaction efficiency strongly depended on the $\mathrm{pH}$ value of the solution. When $\mathrm{pH}$ value was 13.7 , the reaction will complete within $30 \mathrm{~min}$ at $50^{\circ} \mathrm{C}$. However, when the $\mathrm{pH}$ value was 13.0, no nanowires were produced until the reaction temperature was increased to $86^{\circ} \mathrm{C}$. If the $\mathrm{pH}$ value was below 13.0 , the reaction would not take place even at $100^{\circ} \mathrm{C}$. Thus, choosing the proper $\mathrm{pH}$ value is essential on the synthesis of the nickel nanowires as described in this investigation. The important role of $\mathrm{pH}$ value on formation of Ni nanomaterials has also been discussed by other groups $[20,21]$. In addition, we found that higher reaction temperatures will accelerate the reaction processes, that is, it will need less time to complete the reaction at a higher temperature. At the selected reaction temperature of $50{ }^{\circ} \mathrm{C}$ at $\mathrm{pH}$ value of 13.7, the reaction time will be $30 \mathrm{~min}$.

Figure 1a shows the SEM images of nickel nanowires prepared at $50{ }^{\circ} \mathrm{C}, \mathrm{pH}$ value of 13.7 , the strength of the applied magnetic field of $0.5 \mathrm{~T}$ and the concentration of the $\mathrm{Ni}$ ions of $0.08 \mathrm{~mol} / \mathrm{L}$. It can be seen that the nanowires are uniform and the mean diameter is $200 \mathrm{~nm}$ and length of $60 \mu \mathrm{m}$. From the magnified image shown in Fig. 1b, one can see the nanowires are composed of nanoparticles in diameters from 20 to $60 \mathrm{~nm}$. The surfaces of the nanowires are not smooth and there exist a
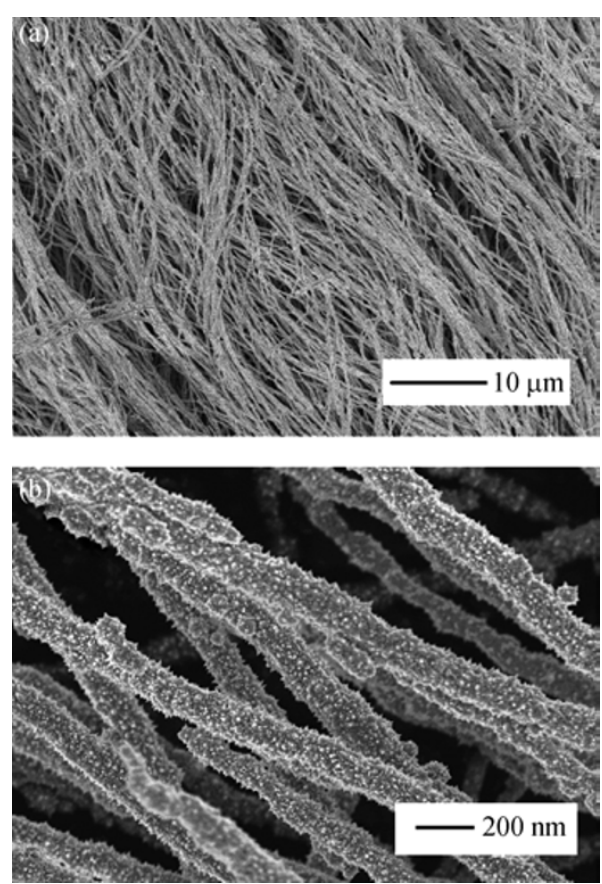

FIG. 1. SEM image of Ni nanowires synthesized via hydrazine hydrate: (a) low magnification image; (b) high magnification image.

lot of spiny-like particles.

The detailed structure of the nanowires was observed by TEM, as shown in Fig. 2a. It is obvious that the Ni nanowires are assembled to 1-D solid linear structure under an applied magnetic field. The magnetic field plays a very important role in the formation of 1-D nanostructure. Even if they were 
ultrasonically treated for 30 minutes, the nickel nanowires will retain their linear structure. This implies that there exist strong magnetic interactions or other interactions among the particles [22]. The selected-area electron diffraction (SAED) pattern (see Fig. 2b) carried on an individual nickel nanowire consists of diffraction rings, indicating that the nickel nanowires have a poly-crystalline structure.

Figure 3 shows the XRD pattern of nickel nanowires. The diffraction peaks of (111), (200) and (220) are consistent with standard diffraction data of nickel (No. JCPDS 04-0850). This indicates that the Ni nanowires are having fcc crystal structure. No other peaks were observed, implying that the nanowires prepared by this method have high purity and they are not oxidized. The lack of oxidation can be attributed to the fact that nitrogen gas was generated during the reaction process. This phenomenon was also observed and discussed by other
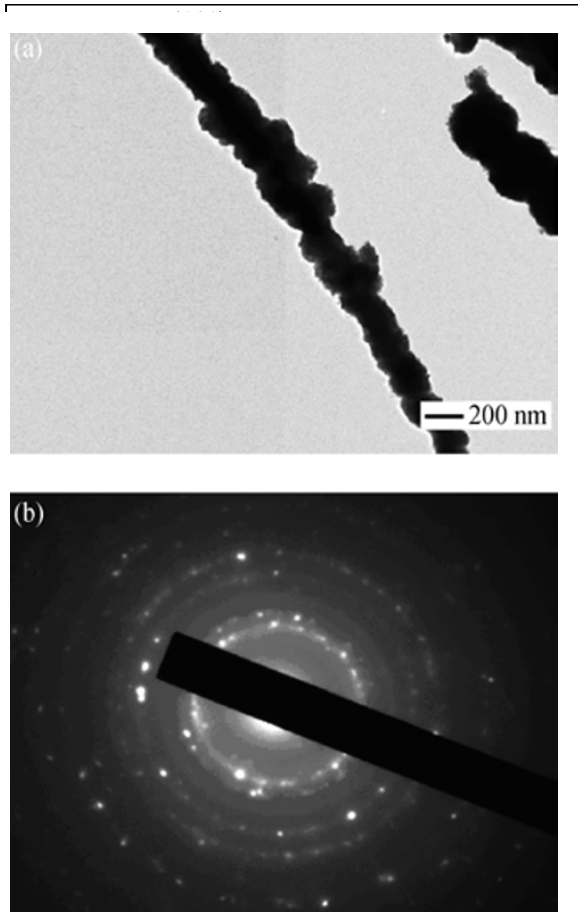

FIG. 2. (a) TEM image of $\mathrm{Ni}$ nanowires. (b) The selected-area electron diffraction (SAED) pattern taken on an individual Ni nanowire.

researchers [18].

Since external magnetic fields played a very important role in formation of 1-D magnetic $\mathrm{Ni}$ nanowires [19,21], we investigated the products prepared without applying magnetic fields. Figure 4 shows the SEM images of the products at $50^{\circ} \mathrm{C}$
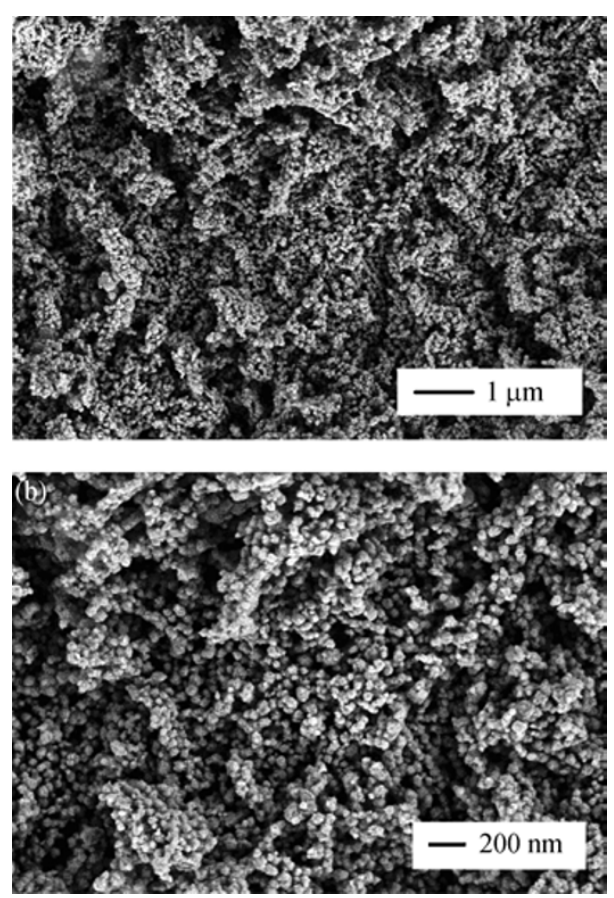

FIG. 4. SEM image of Ni nanoparticles: (a) low-magnification; (b) magnified image.

for $30 \mathrm{~min}$ without magnetic field. One can notice that the products are nanoparticles and without any 1-D nanomaterials present (see Fig. 4a). From the magnified image shown in Fig. $4 \mathrm{~b}$, the size of the particles can be estimated to have a diameter of $100 \mathrm{~nm}$. Therefore, it can be concluded that magnetic field is the prerequisite and mandatory condition to be present in order to grow the magnetic nanowires in this synthetic approach.

In order to illustrate the detailed growth mechanism of the nanowires and the role of magnetic field on the formation of the nanowires, we investigated three samples which were collected during the reaction process. Three samples were collected after the reaction took place for $10 \mathrm{~min}, 20 \mathrm{~min}$ and $30 \mathrm{~min}$. Figure 5 shows that their SEM images and the corresponding schematic illustration of the growth processes of nickel nanowires. One can see at the beginning of the reaction, that is, during the first $10 \mathrm{~min}$ the products are mainly particles with diameter of 100 $\mathrm{nm}$, even if the magnetic field of $0.5 \mathrm{~T}$ was applied. However, in the second $10 \mathrm{~min}$ the particles have been aligned along the magnetic field but the morphology of particles are clear and obvious. After $30 \mathrm{~min}$, the reaction was completed and nanowires with smooth surface were formed. The possible reaction and growth mechanism may be interpreted as follows: 


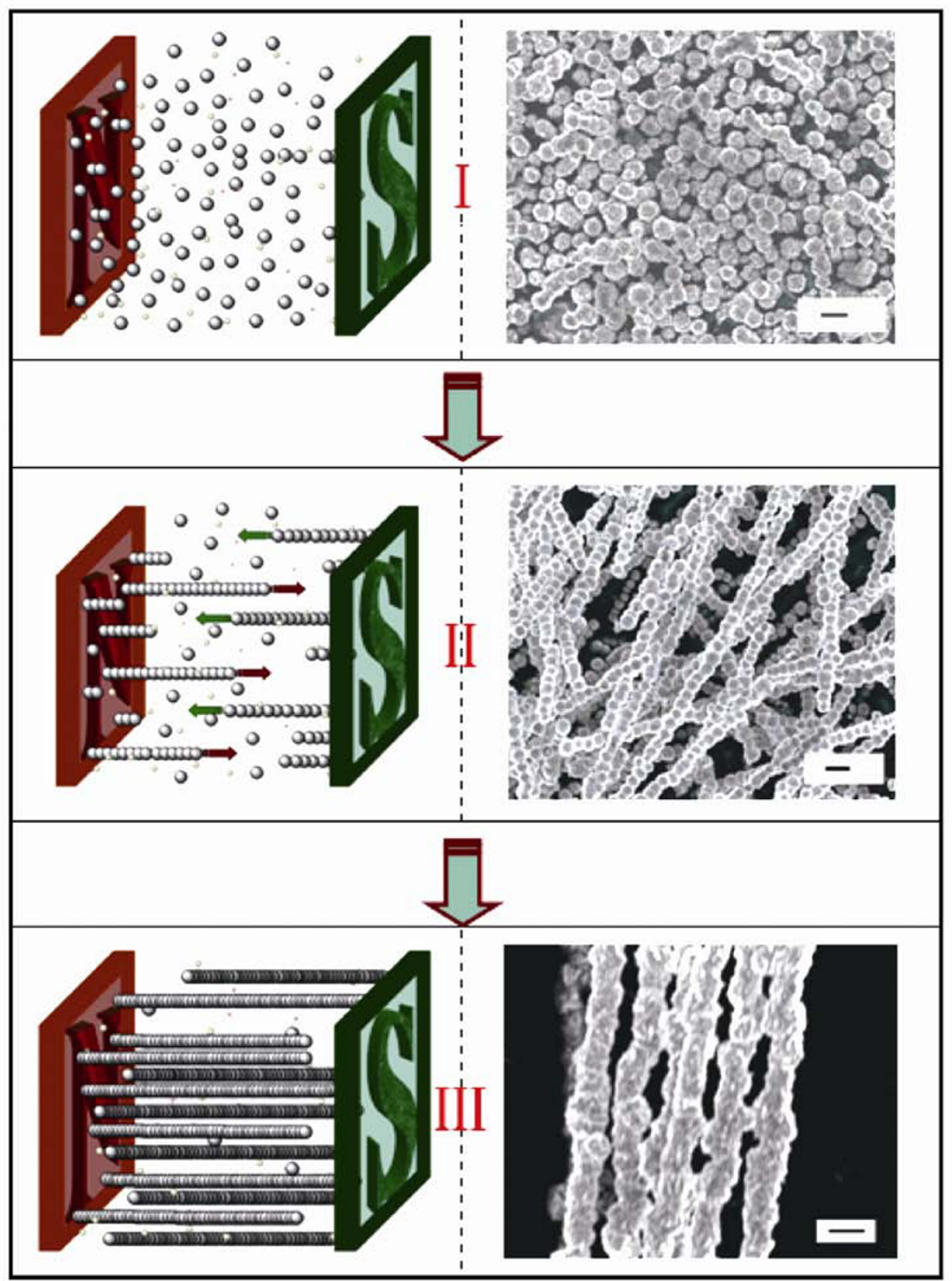

FIG. 5. Schematic illustration of growth mechanism and SEM images of Ni nanowires taken at three steps during the reaction process: (I) after the first 10 min; (II) after the second $10 \mathrm{~min}$; and (III) after the third $10 \mathrm{~min}$. The scale bars in all the insets represent $200 \mathrm{~nm}$.

During the first $10 \mathrm{~min}$, hydrazine hydrate coordinates with $\mathrm{Ni}^{2+}$ to form a very stable complex $\mathrm{Ni}\left(\mathrm{NH}_{3}\right)_{6} \mathrm{Cl}_{2}$, and this would avoid the formation of $\mathrm{Ni}(\mathrm{OH})_{2}$ as $\mathrm{NaOH}$ was introduced into the solution. The formation of $\mathrm{Ni}\left(\mathrm{NH}_{3}\right)_{6} \mathrm{Cl}_{2}$ can greatly decrease the concentration of $\mathrm{Ni}^{2+}$ in the solution and thus can reduce the reaction rate. This will improve the oriented growth of the nickel nuclei and form spherical particles [23]. In the second $10 \mathrm{~min}$, the magnetic particles were subjected to magnetization in the magnetic field and aligned linearly by the magnetic force. The strong magnetic dipole interactions between magnetic particles will make them align tightly and rapidly, forming the magnetic particle-chains. At the same time, the formation of the chains would enhance the local magnetic field [24]. In the third $10 \mathrm{~min}$, new nickel nuclei deposited near the chains as influenced by strong local magnetic field to form linear nanowires as show in Fig. 5c. The magnetic dipole interactions among the particles are so strong, it is difficult to destroy or break down the linear or chain-like structures even as they were subjected to an ultrasonic treatment for $2 \mathrm{~h}$. It must be noted that magnetic dipole interactions among magnetic particles can strongly affect the shape of the nanowires [25]. 


\section{Conclusions}

In summary, nickel nanowires with aspect ratio up to 300 have been successfully prepared by a hydrazine hydrate reduction method, under applied magnetic field. Nickel ions were reduced by the hydrazine hydrate instead of ethanol. The reaction efficiency depends on the $\mathrm{pH}$ value and reaction temperature. A growth mechanism for nanowires is proposed, after investigating the product at three different stages during the synthetic process. The external magnetic field is the key element in the formation of 1-D magnetic nanomaterials, while the strong magnetic dipole interactions among the nanoparticles make the 1-D structure more stable. This work illustrates a facile synthesis of Ni nanowires having high quality. In addition, this synthetic method can be adopted for the preparation of other 1-D magnetic nanomaterials.

This research was supported by the Hi-Tech Research and Development Program of China (No. 2007AA03Z300), Shanghai-Applied Materials Research and Development fund (No. 07SA10), National Natural Science Foundation of China (No. 50730008), Shanghai Science and Technology Grant (No: 0752nm015, 09ZR1414800, 1052nm05500), National Basic Research Program of China (No. 2006CB300406), and the fund of Defence Key Laboratory of Nano/Micro Fabrication Technology.

\section{Received 29 May 2010; accepted 25 June 2010; published online} 15 July 2010

\section{References:}

1. X. G. Peng, L. Manna, W. D. Yang, J. Wickham, E. Scher, A. Kadavanich and A. P. Alivisatos, Nature 404, 59 (2000). doi:10.1038/35003535.

2. S. J. Lei, Z. H. Liang, L. Zhou and K. B. Tang, Mater. Chem. Phys. 113, 445 (2009). doi:10.1016/j.matchemphys. 2008.07.114.

3. H. T. Hu, M. Ouyang, P. D. Yang and C. M. Lieber, Nature 399, 48 (1999). doi:10.1038/19941.

4. D. S. Xu, G. L. Guo, L. L. Gui, Y. Q. Tang, Z. J. Shi, Z. X. Jin, Z. N. Gu, W. M. Liu, X. L. Li and G. H. Zhang, Appl. Phys. Lett. 75, 481 (1999). doi:10.1063/1.124415.

5. C. M. Liu, L. Guo, R. M. Wang, Y. Deng, H. B. Xua and S. H. Yang, Chem. Commun. 2726 (2004). doi:10.1039/ b411311j.

6. N. Vassal, E. Salmon and J. Fauvarque, J. Electrochem. Soc. 146, 20 (1999). doi:10.1149/1.1391558.
7. D. E. Laughlin, B. Lu, Y.-N. Hsu, J. Zou and D. Lambeth, IEEE. Trans. Magn. 36, 48 (2000). doi:10.1109/20.824424.

8. J. M. Richardson and C. W. Jones, J. Mol. Catal. A: Chem. 297, 125 (2009). doi:10.1016/j.molcata.2008.09.021.

9. R.B. Kamble and V. L. Mathe, Sens. Actuators, B: Chem. 131, 205 (2008). doi:10.1016/j.snb.2007.11.003.

10. N. Rezlescu, N. Iftimie, E. Rezlescu, C. Doroftei and P. D. Popa, Sens. Actuators, B: Chem. 114, 427 (2006). doi:10. 1016/j.snb.2005.05.030.

11. Z. Libor and Q. Zhang, Mater. Chem. Phys. 114, 902 (2009). doi:10.1016/j.matchemphys.2008.10.068.

12. S. Thakur, S. C. Katyal and M. Singh, J. Magn. Magn. Mater. 321, 1 (2009). doi:10.1016/i.jmmm.2008.07.009.

13. C. Y. Yu, Y. L. Yu, H. Y. Sun, T. Xu, X. H. Li, W. Li, Z. S. Gao and X. Y. Zhang, Mater. Lett. 61, 1859 (2007). doi:10.1016/j.matlet.2006.07.162.

14. H. J. Zhang and Y. Liu, J. Alloys Compd. 458, 588 (2008). doi:10.1016/j.jallcom.2007.05.016.

15. Y. G. Sun and Y. N. Xia, Adv. Mater. 14, 833 (2002). doi:10.1002/1521-4095(20020605)14:11<833::AI D-ADMA833>3.0.CO;2-K.

16. H. L. Niu, Q. W. Chen, M. Ning, Y. S. Jia and X. J. Wang, J. Phys. Chem. B 108, 3996 (2004). doi:10.1021/jp0361172.

17. C. H. Gong, L. G. Yu, Y. P. Duan, J. T. Tian, Z. S. Wu and Z. J. Zhang, Eur. J. Inorg. Chem. 18, 2884 (2008). doi:10.1002/ejic.200800200.

18. L. Y. Zhang, J. Wang, L. M. Wei, P. Liu, H. Wei and Y. F. Zhang, Nano-Micro Letters 1, 49 (2009). doi:10.5101/ nml.v1i1.p49-52.

19. P. Liu, Z. J. Li, B. L. Yadian and Y. F. Zhang, Mater. Lett. 63, 1650 (2009). doi:10.1016/j.matlet.2009.04.031.

20. S.H. Wu and D.H. Chen, J. Colloid Interface Sci. 259, 282 (2003). doi:10.1016/S0021-9797(02)00135-2.

21. C. H. Gong, J. T. Tian, T. Zhao, Z. S. Wu and Z. J. Zhang, Mater. Res. Bull. 44, 35 (2009). doi:10.1016/j.materresbull. 2008.04.010.

22. M. Zhang, J. Deng, M. H. Zhang and W. Li, Chinese J. Catal. 30, 447 (2009). doi:10.1016/S1872-2067(08) 60111-4.

23. Z. Liu, S. Li, Y. Yang, S. Peng, Z. Hu and Y. Qian, Adv. Mater. 15, 1946 (2003). doi:10.1002/adma.200305663.

24. E. K. Athanassiou, P. Grossmann, R. N. Grass and W. J. Stark, Nanotechnology 18, 165606 (2007). doi:10.1088/ $\underline{0957-4484 / 18 / 16 / 165606 .}$.

25. Y. Hou, S. Gao, T. Ohta and H. Kondoh, Eur. J. Inorg. Chem. 4, 1169 (2004). doi:10.1002/ejic.200300779. 